\title{
Ethical challenges in tracheostomy-assisted ventilation in amyotrophic lateral sclerosis
}

\author{
Morten Magelssen ${ }^{1}\left(\right.$ - Trygve Holmøy $y^{2,3} \cdot$ Morten Andreas Horn $^{4} \cdot$ Ove Arne Fondenæs ${ }^{5} \cdot$ Knut Dybwik $^{6,7}$. \\ Reidun Førde ${ }^{1}$
}

Received: 25 June 2018 / Revised: 7 September 2018 / Accepted: 7 September 2018 / Published online: 14 September 2018

c) Springer-Verlag GmbH Germany, part of Springer Nature 2018

\begin{abstract}
The special nature of amyotrophic lateral sclerosis (ALS) and tracheostomy with invasive ventilation (TIV) leads to challenges that can be difficult in two senses: not only to handle well, but also to discuss with patients and other involved stakeholders. Because of the delicate nature of interpersonal relations and communication in ALS, some of the downsides to TIV may almost take on a nature of taboo, making them difficult to raise for open discussion. Yet these ethical challenges are important to be aware of, not only for health professionals and managers but, arguably, also for patients and next of kin. They are important also for a wider professional and societal debate about whether and to whom TIV should be offered. In this paper we highlight and examine ethical challenges in TIV for ALS, with a special emphasis on those that are hard to discuss openly and that therefore might fail to be addressed. The analysis is structured by the four core principles of healthcare ethics: beneficence, nonmaleficence, respect for patient autonomy, and justice.
\end{abstract}

Keywords Amyotrophic lateral sclerosis $\cdot$ Ethics $\cdot$ Beneficence $\cdot$ Invasive ventilation $\cdot$ Patient autonomy $\cdot$ Tracheostomy

\section{Introduction}

Today most untreated ALS patients die from respiratory failure within 3 years of diagnosis [1]. Non-invasive ventilation (NIV) relieves symptoms and prolongs life, especially in patients with non-bulbar ALS [2-5]. However, in most cases non-invasive ventilation will eventually become difficult to

Morten Magelssen

morten.magelssen@medisin.uio.no

1 Centre for Medical Ethics, Institute of Health and Society, University of Oslo, PB 1130, Blindern, 0318 Oslo, Norway

2 Department of Neurology, Akershus University Hospital, Lørenskog, Norway

3 Institute of Clinical Medicine, University of Oslo, Oslo, Norway

4 Department of Neurology, Oslo University Hospital, Oslo, Norway

5 National Advisory Unit on Longterm Mechanical Ventilation, Haukeland University Hospital, Bergen, Norway

6 Department of Anaesthesia and Intensive Care Medicine, Nordland Hospital, Bod $\emptyset$, Norway

7 Nord University, Bodø, Norway tolerate or manage as bulbar function deteriorates. Weakness in the face or upper airway muscles may in some cases make it impossible to introduce NIV in the first place [6]. For these patients, invasive mechanical ventilation with tracheostomy (TIV) is the only alternative to death when respiratory failure is manifest.

The use of TIV in ALS patients is challenging because it allows the patient to survive with increasing pareses, which will render him or her totally dependent on help from others and ultimately may lead to a locked-in state with the inability to communicate. Whereas the ventilator is itself rather inexpensive, the economic, social and emotional burdens of 24/7 care are challenging both to formal and informal caregivers [7]. The use of TIV in ALS varies ranging from $0 \%$ of patients from the UK, $1-14 \%$ in the USA, 3\% in Germany, $2-5 \%$ in France, $11 \%$ in northern Italy, to $27-45 \%$ in Japan, likely reflecting cultural, economic, legal and organisational differences both within and outside the healthcare system $[8$, 9]. In Norway and Sweden, $6.7 \%$ of men and $3.8 \%$ of women living with ALS between 2002 and 2007 used TIV, suggesting that gender may also influence preferences or access to such treatment [10].

The use of TIV in ALS patients thus raises several ethical issues. Alongside the benefits of treatment may come 
negative consequences for both patient, next of kin, health professionals, healthcare systems and society. Because of disease-specific factors, but most of all because of the prospect of death and the delicate nature of interpersonal relations and communication in ALS, some of the downsides to TIV may almost take on a nature of taboo, making them difficult to raise for open discussion among the different stakeholders. Yet, these ethical challenges are important to be aware of, for health professionals, managers and, arguably, also for patients and next of kin. They are important also for a wider professional and societal debate about whether and to whom TIV should be offered.

The aim of this paper is to bring to light and examine ethical challenges with TIV in patients with ALS, with a special emphasis on what we perceive as the most difficult challenges. Our analysis draws on clinical experience from Norway, in addition to published research. Societal contexts and medical cultures differ. In the Scandinavian countries, patients do not have an unqualified right to TIV; the responsible physician has the formal decision-making authority. Although some of the arguments we make might take on a different character or be accorded less weight in other medical cultures, most of the arguments are likely to be of some relevance also in medical cultures that differ from the Scandinavian. Of note, our analysis under the heading 'justice' pertains specifically to the context of publicly funded healthcare systems such as those of many European countries. The analysis is structured by the four core principles of healthcare ethics: beneficence, nonmaleficence, respect for patient autonomy, and justice.

\section{Ethical challenges}

\section{Beneficence}

A basic question to ask about TIV for ALS is whether and to what extent it is a beneficial intervention. ALS is a progressive, incurable, fatal disease. The prognosis depends on the individual disease course, but nutritional and respiratory status will influence life-expectancy. The main goal of treatment is to minimise morbidity and maximise quality of life (QoL) although some patients might perceive maximising life-expectancy as a goal in itself.

How much TIV actually prolongs life appears to vary hugely between countries, reflecting both differing medical practices, legislation, social/religious and cultural issues as well as provision and organisation of caregiving and financial considerations. Median survival in uncontrolled groups varies from 8 to 89 months in the literature [11-14]. Age $<65$ years, marital status, previous NIV, the option to receive care in the home and rapid deterioration of respiratory function may promote a choice of TIV. Some patients become dissatisfied over time; the most common reason for patient requests for the withdrawal of TIV in end-stage ALS is loss of meaning of life [15]. Before commencing treatment, it can be difficult to predict how treatment and QoL will be perceived by the particular patient. This must be communicated to the patient prior to deciding to start TIV.

Several studies have shown that QoL is maintained with disease progression and is not dependent on physical function, but rather relies more upon psychological and existential factors [16-19]. In general, assessing the quality of life of another person requires caution. In patients with severe diseases, such as ALS, both healthcare professionals, caregivers and healthy individuals seem to underestimate not only QoL, but also other patient-perceived metrics [20-23]. To many, the plight of the ALS sufferer, experiencing relentless gradual loss of function with a total locked-in syndrome (TLS) as the potential end state, appears to be one of the worst fates imaginable. Yet it has been demonstrated that patients who suffer a dramatic loss of function often undergo a reorientation, coming to terms more or less with their new situation of disability [24, 25].

However, interpretation of reported patient satisfaction with the choice of life-supportive treatment is by nature difficult, when death is the only alternative to the intervention. Perhaps controversially, based on clinical experience we claim that subtle psychological mechanisms might come into play when the patient is asked to report their satisfaction. For instance, acknowledging one's ambivalence or outright regret in having chosen TIV can be hard: the patient will then have to live not only with the consequences of a regrettable choice, but also with guilt or other negative emotions that come from having placed oneself and one's loved ones in a difficult situation. In our view, decision-making cannot be based solely upon reported satisfaction [26].

\section{Nonmaleficence}

A negative consequence of initiating TIV is that death may then only ensue either from an explicit choice, or from an unrelated fatal illness or accident [27]. When adequate nutrition and comprehensive, competent caregiving is provided the ventilator may prolong life considerably. There might then no longer be an opportunity to elect to 'let nature take its course', dying solely from the disease itself, and not also in some measure from the decision to withdraw treatment, be it the patient's or the physician's. The disease is an ultimate cause of death, but the decision to discontinue TIV is also (in some sense) a cause of death. This situation can place an additional burden on the patient as well as the surroundings.

Patients considering TIV must be informed of the right to have the treatment withdrawn, and also that expressing a wish for treatment withdrawal may become increasingly difficult throughout the disease course. Problems are most 
likely to arise where there has been no prior discussion and agreement on criteria for when TIV should be withdrawn, or when it is unclear whether the patient later on has changed his or her mind. It is, therefore, essential to carefully discuss and decide on such criteria together with the patient prior to commencing TIV. However, a prior agreement on withdrawal criteria does not solve every problem with the proper timing of withdrawal. Particularly if these are based on poorly defined criteria for motor function such as entering the locked-in state (TLS), there is a substantial risk of harm that stems from the problem of getting the timing of the discontinuation of TIV right. In general, if TIV is halted too soon, then the patient loses valuable time; if too late, then the patient is burdened with a prolonged dying process and time wherein the burdens of treatment and of the condition are not made up for by the benefit of living. The timing is demanding because deterioration is gradual and there might be no natural and readily identifiable stage where TIV ought to be discontinued. Communication with the patient about preferences at this stage might be exceedingly hard; first, because of difficulties from the advanced paralysis in the end-stage of disease; second, because of previously unrecognised and developing cognitive/executive dysfunction that occur in a substantial proportion of patients, and that might be difficult to assess. For instance, although in theory the cessation of the ability to communicate through eye movements is a clear demarcation line, in practice there might be a phase in which the patient's communication is variable and/or reduced, and clinicians become unsure of whether the relevant threshold has been reached. If the clinician is to be on the safe side-'erring on the side of life'—one risks overtreatment in a phase in which quality of life is poor. This would constitute a substantial harm.

In our view, when the timing is right the discontinuation of TIV is medically and ethically proper. The intention is to spare the patient of continued treatment which has been found to no longer be of benefit, and the intention is thus morally upright. Laws, mores and cultural traditions that obstruct withdrawal of life-prolonging treatment when continued treatment is not in the patient's interest contribute to overtreatment, leading to both patient harm and waste of healthcare resources.

In medical ethics, the principle of nonmaleficence pertains to risks, harms and other negative consequences of healthcare for the patient. However, because TIV in ALS affects not only the patient but certainly also the next of kin and the professional caregivers, it is highly relevant to discuss negative consequences of treatment also for these other stakeholders [28]. Indeed, TIV is an invasive treatment in more than one sense of the word. Particularly when provided in the patient's home, TIV transforms many aspects of the family's daily life. For the next of kin there are not only benefits but also potentially very significant burdens.
Arguably, therefore, the traditional paradigm wherein the views of the next of kin are of little significance when the patient remains formally competent is challenged by the case of TIV for ALS.

The obvious benefit for the next of kin is that their loved one remains alive for extra months or years. The burdens, however, are subtler and might be particularly challenging to talk about in an open manner. Although TIV extends life it also postpones death; the patient remains alive in a severely disabled state, which prevents the next of kin from grieving and moving on with their lives. In some situations, this might be of particular significance for any underage children. What does it do to them to have their parent 'locked' into such a state with, in particular, reduced means of communication for an indefinite time? For a spouse, divorce or separation might be perceived as cruel and morally impossible, thus leaving no choice but to support the patient in the decision to choose and to continue TIV. In a German study the QoL of ALS patients on NIV and TIV was compared to that of their caregivers, mainly spouses. The study showed a good overall QoL for both NIV and TIV patients, but a very high burden of care for TIV caregivers, $30 \%$ of whom rated their own QoL lower than that of the patient. Sexuality was an important issue. The authors conclude that in the assessment of QoL in a home palliative care situation the primary caregivers should always be included [29].

Knowing that a proportion of ALS patients experience early cognitive decline, there is also the somewhat disturbing — but nonetheless important—question of to what degree the patient is able to empathise with the plight of the next of kin. How does the patient take the interests of the next of kin into account when deciding for or against the commencement or continuation of TIV? In our view, inability or unwillingness of the patient to reflect upon the likely negative impact of caregivers' QoL should be taken into consideration when deciding for or against offering TIV. At the very least, the burden on spouse and children should be a compulsory part of the information given before the choice of TIV is taken. Irrespective of this there is another subtle moral problem: The patient might, by their very existence, burden their loved ones, and be aware of being a burden. Is it reasonable to expect the patient to take into account the burdens to their next of kin when deciding for or against TIV, when their own life is at stake?

The very discussion of whether TIV should be commenced can put the next of kin in an awkward situation. Despite the clear intention of physicians that next of kin should neither encourage or discourage, but support the patient in decision-making, next of kin may feel obliged to promote the prolongation of life. In a sense, how can they do anything but encourage the patient to choose TIV? Anything less could risk implying that the patient's death would come as a welcome relief. Health professionals should be aware of 
such complicated interpersonal dynamics that are put into play when TIV is up for discussion.

Providing care for TIV patients in their own home represents great challenges for the healthcare professionals (HCPs) involved. Many HCPs find their status as mere guests in the patients' homes difficult. Their decisions might be challenged by family members who have become experts on the patient's care, and it might be impossible to satisfy the expectations of both administrators, patient and family members. Such challenges can lead to professional dissatisfaction, moral distress and burnout, and high turnover rates, leading to lack of continuity and competence among HCPs [30].

\section{Respect for patient autonomy}

Respect for the patient's right to accept or refuse medical interventions are mainstays of modern healthcare. Prerequisites, however, are competence and being informed. Whether these requirements are in place might sometimes be questioned in cases of TIV for ALS. Cognitive and behavioural impairment is highly relevant to patient autonomy and decision-making. ALS-specific changes may be subtle, or they may be overt, such as in frontotemporal dementia (FTD). The overlap between ALS and FTD is well established on a neuropathological, genetic and clinical level. In recent publications, estimates of up to one-half of the patient population demonstrates heterogeneous changes in cognition or behaviour, $15 \%$ meeting the criteria for FTD [31]. The need to assess routinely for such manifestations, both prior to critical decisions and possibly during the course of the treatment, is comparable to assessing the degree of competence, a defining feature of patient autonomy. Ultimately, such assessments may be crucial to achieve real empowerment and genuinely shared decision-making. Recent studies suggest that the development of cognitive impairment follows a rather specific pattern as the disease process spreads across the brain [32]. Thus, the level of cognitive impairment and autonomy may change throughout the disease process, and evaluations performed before initiation of TIV may not be relevant later on.

The diverse aspects of cognitive impairment and behavioural abnormalities that may be seen in ALS patients include the following: loss of executive functions, loss of ability to change focus, inexplicable deviations from previously stated opinions, loss of insight, loss of empathy, in addition to problems with speech and thinking that compound communication problems due to dysarthria. These are among the very skills that a patient needs to wield their autonomy and to display decision-making competence. How does one resolve issues of autonomy and competence in a patient that is so dramatically bereft of the tools of autonomy, and might lack the communication skills to prove their competence?

If an ALS patient becomes fully dependent on the assistance of their family to realise the slightest acts of autonomy - is the patient then really autonomous? What degree of insight and empathy might one expect from a patient in such a situation? The kind of symbiosis that develops between ALS patients and their closest caregivers challenges the traditional view on patient autonomy. Even though this peculiar state that ALS patients and their caregivers frequently reach is well-known to HCPs caring for ALS patients, it is quite different from most experiences we usually have as human beings, and as patients with less incapacitating disorders. Is it possible for a patient who is not yet there, to truly envision such a state? How does the cognitive impairment seen in ALS impact on the ability to perform such thought experiments? And even if the patient is able, is it reasonable to demand that they undertake the weighing of their own interests-which include the ability to go on living — against the burden placed on their loved ones? What does an informed choice imply in such a situation-what should the patient be required to take into consideration before the choice is sufficiently informed?

In our experience, most ALS patients are relieved when given the chance to talk with health professionals about the terminal stages of their disease. Nevertheless, some patients seem uncomfortable discussing whether to seek TIV as the disease progresses. Apparently, these patients prefer not to think about the subject, and are reluctant to make active choices about their own death. They may, however, feel pressured into doing so because the health professionals need to clarify what to do when respiratory failure ensues. In this way, the very availability of TIV places a burden on some patients: Their right to withdraw from psychologically challenging (perhaps even damaging) discussions and choices about TIV, the end stages of the disease and death, may then be denied them [33].

\section{Justice}

Fair priority setting in healthcare has become a virtue of necessity even in affluent countries. In general, resources spent on treatment $A$ for patient group $X$ could instead have been spent on treatment B for patient group Y. Both clinicians and politicians are sometimes reluctant to perform overt rationing, especially when identifiable patients are thereby denied beneficial services [34]. Yet certain healthcare services are exceedingly resource intensive and/ or provide relatively meagre benefits. Clinicians and other decision-makers need to increase their awareness of such issues if priority setting is to be performed in a way that promotes equitable distribution of scarce resources. 
In this context, TIV for ALS is a very resource intensive service. Few studies examine the cost of home mechanical ventilation. In a recent Canadian study, however, the overall median monthly healthcare cost with invasive ventilation was $€ 6,200$ across all diagnoses with the highest cost in ALS (range $€ 6031-12,471$ ) [35]. In a Norwegian context the presence of professional carers in the patient's home around the clock would incur yearly costs in excess of $€ 500,000$, which would be covered by public funds. Even when the varying costs between countries are taken into account, the cost-perQALY (quality-adjusted life year) for TIV for ALS appears to be very high, implying a low cost-effectiveness compared to most other healthcare services. Advocates for fair priority setting in healthcare by way of cost-effectiveness calculations typically maintain that a condition for fairness is that services are treated similarly across specialties and sectors. Thus, the principle of equality might appear to entail that TIV for ALS is too expensive for the benefit it confers, and thus ought not to be offered within public healthcare services. The resources might be better spent on other treatments for other patients with other conditions, as more health is generated thereby.

However, there are at least three important reasons to resist this conclusion. First, TIV is offered for many conditions other than ALS. It is unreasonable to consider a proposal to defund TIV only for one of these conditions, if treatment is comparably costly for the others. Second, the state might finance treatment that is even much less costeffective than TIV for ALS. Third, in most countries there is no explicit threshold for acceptable cost-effectiveness that has been adopted by the state for the entire healthcare sector. Sanctioning by the highest level of democratic government would be necessary to confer legitimacy on such a threshold. Arguably, it is not reasonable to expect the professional community itself to decide to cease offering certain services due to resource constraints and poor cost-effectiveness. Any decision to limit the offer of TIV for ALS patients would be hard to make, and could also be felt to go against the clinician's primary commitment to the good of his or her patients [36]. Any such new policy, then, should either be made at the political level where the relevant authority resides, or, alternatively, follow explicitly and unambiguously from general priority setting principles adopted at the political level. As long as the relevant political support is lacking, discontinuing TIV for ALS patients merely because of low cost-effectiveness arguably is not justified.

\section{The need for good decision-making processes}

The ethical challenges examined here substantiate the claim that treatment with TIV is not necessarily in the best interests of the ALS patient or the next of kin, even when the patient expresses a preference for it. There are substantial risks_-risks of making a choice that will later be regretted, of overtreatment and having to endure poor quality of life due to improper timing of treatment withdrawal, and of burdening next of kin. As shown, in a traditional medicoethical «four principles» framework, the next of kin and their plight risk being neglected; yet their interests should also be safeguarded. Similarly, the interests of healthcare professionals and carers have little place in the framework, yet their viewpoints should also be taken into consideration.

In our view, the presence of these risks places great demands on the quality of the decision-making process [33]. Physicians must recognize the responsibility that comes with their great power to structure and set the agenda for the decision-making process. The patient's right to remain undecided must be respected, even though this might lead to further dilemmas. In our experience, some patients do not want to make a choice about TIV in spite of repeated attempts to address the issue. In some cases, such patients are later on resuscitated from an acute $\mathrm{CO}_{2}$ narcosis. They may then have to choose between TIV and death-the same question that they previously did not want to answer or in some cases even discuss. Patients should be confronted with such a scenario, including the possibility that HCPs might refrain from future resuscitation procedures as long as the patient refrains from making a decision regarding TIV.

\section{Conclusion}

The main message of this article has been that the special nature of ALS and of TIV leads to a set of challenges that are more or less specific to these treatment choices, and that are difficult in two senses: First, they might be difficult to handle well; second, and more interestingly, they might be difficult to discuss, however well aware clinicians are of them. Problems such as future prospects for treatment withdrawal and the balancing of the interests of patient and next of kin, respectively, might be difficult to discuss openly in the physician-patient setting. Problems such as the low costeffectiveness of the treatment, and the plight of next of kin can be difficult to communicate to the public. Finally, problems such as the demanding work situation for professional carers in the patient's home and the burdens on next of kin are-because they do not touch on the patient's interests directly-difficult to give weight in decisions and guideline documents. To improve decision-making, health professionals should discuss more of these challenges openly, with the requisite sensitivity. Criteria for decision-making can be of some help, yet a great deal of discretion is required for the clinicians engaged in the decision of whether treatment should be offered. 


\section{Compliance with ethical standards}

Conflicts of interest Dr. Magelssen has received a fee for a lecture for Sanofi-Aventis. Dr. Holmøy has received unrestricted research grants from The Norwegian ALS foundation.

Ethical standards Ethics approval was not required for this study as it did not involve the generation of new data.

\section{References}

1. Rooney J, Byrne S, Heverin M, Corr B, Elamin M, Staines A et al (2013) Survival analysis of Irish amyotrophic lateral sclerosis patients diagnosed from 1995 to 2010. PLoS One 8(9):e74733

2. Miller RG, Jackson CE, Kasarskis EJ, England JD, Forshew D, Johnston W et al (2009) Practice parameter update: the care of the patient with amyotrophic lateral sclerosis: drug, nutritional, and respiratory therapies (an evidence-based review): report of the Quality Standards Subcommittee of the American Academy of Neurology. Neurology 73(15):1218-1226. https://doi. org/10.1212/WNL.0b013e3181bc0141

3. Andersen PM, Abrahams S, Borasio GD, de Carvalho M, Chio A, Van Damme P et al (2012) EFNS guidelines on the clinical management of amyotrophic lateral sclerosis (MALS) - revised report of an EFNS task force. Eur J Neurol 19(3):360-375. https ://doi.org/10.1111/j.1468-1331.2011.03501.x

4. Radunovic A, Annane D, Rafiq MK, Mustfa N (2013) Mechanical ventilation for amyotrophic lateral sclerosis/motor neuron disease. Cochrane Database Syst Rev 10:CD004427

5. Berlowitz DJ, Howard ME, Fiore JF, Vander Hoorn S, O'Donoghue FJ, Westlake J et al (2016) Identifying who will benefit from non-invasive ventilation in amyotrophic lateral sclerosis/ motor neurone disease in a clinical cohort. J Neurol Neurosurg Psychiatry 87(3):280-286

6. Connolly S, Galvin M, Hardiman O (2015) End-of-life management in patients with amyotrophic lateral sclerosis. Lancet Neurol 14(4):435-442

7. Lerum SV, Solbrække KN, Frich JC (2017) Healthcare professionals' accounts of challenges in managing motor neurone disease in primary healthcare: a qualitative study. Health Soc Care Community 25(4):1355-1363

8. Weber C, Fijalkowska B, Ciecwierska K, Lindblad A, BaduraLotter G, Andersen PM et al (2017) Existential decision-making in a fatal progressive disease: how much do legal and medical frameworks matter? BMC Palliat Care 16(1):80

9. Dybwik K, Tollåli T, Nielsen EW, Brinchmann BS (2010) Why does the provision of home mechanical ventilation vary so widely? Chronic Respir Dis 7(2):67-73

10. Tollefsen E, Midgren B, Bakke P, Fondenes O (2010) Amyotrophic lateral sclerosis: gender differences in the use of mechanical ventilation. Eur J Neurol 17(11):1352-1357

11. Chio A, Calvo A, Ghiglione P, Mazzini L, Mutani R, Mora G et al (2010) Tracheostomy in amyotrophic lateral sclerosis: a 10-year population-based study in Italy. J Neurol Neurosurg Psychiatry 81(10):1141-1143. https://doi.org/10.1136/jnnp.2009.175984

12. Tagami M, Kimura F, Nakajima H, Ishida S, Fujiwara S, Doi Y et al (2014) Tracheostomy and invasive ventilation in Japanese ALS patients: decision-making and survival analysis: 19902010. J Neurol Sci 344(1-2):158-164. https://doi.org/10.1016/j. jns.2014.06.047

13. Dreyer P, Lorenzen CK, Schou L, Felding M (2014) Survival in ALS with home mechanical ventilation non-invasively and invasively: a 15-year cohort study in west Denmark. Amyotroph
Lateral Scler Frontotemporal Degener 15(1-2):62-67. https:// doi.org/10.3109/21678421.2013.837929

14. Heritier Barras AC, Adler D, Iancu Ferfoglia R, Ricou B, Gasche Y, Leuchter I et al (2013) Is tracheostomy still an option in amyotrophic lateral sclerosis? Reflections of a multidisciplinary work group. Swiss Med Wkly 143:w13830. https://doi. org/10.4414/smw.2013.13830

15. Dreyer PS, Felding M, Klitnæs CS, Lorenzen CK (2012) Withdrawal of invasive home mechanical ventilation in patients with advanced amyotrophic lateral sclerosis: ten years of Danish experience. J Palliat Med 15(2):205-209

16. Paganoni S, Karam C, Joyce N, Bedlack R, Carter GT (2015) Comprehensive rehabilitative care across the spectrum of amyotrophic lateral sclerosis. NeuroRehabilitation 37(1):53-68. https ://doi.org/10.3233/NRE-151240

17. Calvo A, Moglia C, Ilardi A, Cammarosano S, Gallo S, Canosa A et al (2011) Religiousness is positively associated with quality of life of ALS caregivers. Amyotroph Lateral Scler 12(3):168-171. https://doi.org/10.3109/17482968.2011.560947

18. Chio A, Gauthier A, Montuschi A, Calvo A, Di Vito N, Ghiglione P et al (2004) A cross sectional study on determinants of quality of life in ALS. J Neurol Neurosurg Psychiatry 75(11):1597-1601. https://doi.org/10.1136/jnnp.2003.033100

19. Simmons Z, Bremer BA, Robbins RA, Walsh SM, Fischer S (2000) Quality of life in ALS depends on factors other than strength and physical function. Neurology 55(3):388-392

20. Slevin ML, Plant H, Lynch D, Drinkwater J, Gregory WM (1988) Who should measure quality of life, the doctor or the patient? Br J Cancer 57(1):109-112

21. Kubler A, Winter S, Ludolph AC, Hautzinger M, Birbaumer N (2005) Severity of depressive symptoms and quality of life in patients with amyotrophic lateral sclerosis. Neurorehabilit Neural Repair 19(3):182-193. https://doi.org/10.1177/15459 68305276583

22. Lule D, Ehlich B, Lang D, Sorg S, Heimrath J, Kubler A et al (2013) Quality of life in fatal disease: the flawed judgement of the social environment. J Neurol 260(11):2836-2843. https:// doi.org/10.1007/s00415-013-7068-y

23. Rothwell PM, McDowell Z, Wong CK, Dorman PJ (1997) Doctors and patients don't agree: cross sectional study of patients' and doctors' perceptions and assessments of disability in multiple sclerosis. BMJ 314(7094):1580-1583

24. Rousseau MC, Pietra S, Blaya J, Catala A (2011) Quality of life of ALS and LIS patients with and without invasive mechanical ventilation. J Neurol 258(10):1801-1804. https://doi. org/10.1007/s00415-011-6018-9

25. Bruno M-A, Bernheim JL, Ledoux D, Pellas F, Demertzi A, Laureys S (2011) A survey on self-assessed well-being in a cohort of chronic locked-in syndrome patients: happy majority, miserable minority. BMJ Open 1(1):e000039

26. Rousseau MC, Baumstarck K, Billette de Villemeur T, Auquier $P$ (2016) Evaluation of quality of life in individuals with severe chronic motor disability: a major challenge. Intractable Rare Dis Res 5(2):83-89. https://doi.org/10.5582/irdr.2016.01017

27. Lerum SV, Solbrække KN, Holmøy T, Frich JC (2015) Unstable terminality: negotiating the meaning of chronicity and terminality in motor neurone disease. Sociol Health Illn 37(1):81-96

28. Dybwik K, Tollåli T, Nielsen EW, Brinchmann BS (2011) "Fighting the system": families caring for ventilator-dependent children and adults with complex health care needs at home. BMC Health Serv Res 11(1):156

29. Kaub-Wittemer D, Steinbuchel N, Wasner M, Laier-Groeneveld G, Borasio GD (2003) Quality of life and psychosocial issues in ventilated patients with amyotrophic lateral sclerosis and their caregivers. J Pain Symptom Manag 26(4):890-896 
30. Dybwik K, Nielsen EW, Brinchmann BS (2011) Home mechanical ventilation and specialised health care in the community: between a rock and a hard place. BMC Health Serv Res 11:115. https://doi. org/10.1186/1472-6963-11-115

31. Woolley SC, Rush BK (2017) Considerations for clinical neuropsychological evaluation in amyotrophic lateral sclerosis. Arch Clin Neuropsychol 32(7):906-916. https://doi.org/10.1093/arcli n/acx089

32. Lulé D, Böhm S, Müller H-P, Aho-Özhan H, Keller J, Gorges M et al (2018) Cognitive phenotypes of sequential staging in amyotrophic lateral sclerosis. Cortex 101:163-171

33. Oliver DJ, Turner MR (2010) Some difficult decisions in ALS/ MND. Amyotroph Lateral Scler 11(4):339-343. https://doi. org/10.3109/17482968.2010.487532
34. Menzel P (2014) Statistical versus indentified lives. Why not to use the "R" word. In: Danis M, Hurst S, Fleck L, Førde R, Slowther A (eds) Fair resource allocation and rationing at the bedside. Oxford University Press, New York, pp 238-252

35. Nonoyama ML, McKim DA, Road J, Guerriere D, Coyte PC, Wasilewski $\mathrm{M}$ et al (2018) Healthcare utilisation and costs of home mechanical ventilation. Thorax. https://doi.org/10.1136/ thoraxjnl-2017-211138

36. Magelssen M, Nortvedt P, Solbakk JH (2016) Rationing at the bedside: immoral or unavoidable? Clin Ethics 11(4):112-121. https://doi.org/10.1177/1477750916657664 